\title{
Evaluation of the Influence of Anomalies on the Flow of Non-Newtonian Fluid in Carotid Bifurcation by the PIV Method
}

\author{
Darina Jasikova ${ }^{1, *}$, Nikola Pluchova ${ }^{2}$, Michal Kotek ${ }^{1}$, and Václav Kopecký1 \\ ${ }^{1}$ Department of Physical Measurement, Technical University of Liberec, Institute for Nanomaterials, \\ Advanced Technology and Innovation, Studentska 1402/2, Liberec 1, 461 17, Czech Republic \\ ${ }^{2}$ Faculty of Health Studies, Technical University of Liberec, Institute for Nanomaterials, Advanced \\ Technology and Innovation, Studentska 1402/2, Liberec 1, 461 17, Czech Republic
}

\begin{abstract}
The blood flow in anomalous carotid bifurcation is associated with stroke. Changed character of the flow, especially the occurrence of vortex structures, has showed a high correlation with thrombus formation and the development of atherosclerotic changes. The purpose of this work is to perform comparative model measurements of the flow inside the internal carotid bifurcation and to evaluate the influence of the geometry stenosis, aneurysm on changes in the interaction of the fluid with the wall surface, i.e. on the local hemodynamic. The study uses the Global Imaging Methods for visualization and measurement of the phantom blood flow. The evaluation of the measured dataset and their subsequent analysis confirms the impact on the origin and development of pathological changes.
\end{abstract}

\section{Introduction}

The blood flow in anomalous carotid bifurcation is associated with stroke. Stroke is the third most common cause of death in developed countries. Carotid stenosis is responsible for more than $25 \%$ of all ischemic strokes. The carotid artery is prone to aneurysms and the deposition of atherosclerotic plaques. It is caused by atherosclerotic degeneration, fibromuscular dysplasia or trauma. One third of ischemic strokes are caused by atherosclerotic carotid bifurcation. Only $15 \%$ of stroke victims are warned by a transient ischemic attack and waiting until the first symptoms is risky. Therefore, early detection and prevention of stroke is important.

Nowadays, there are used many methods to examine carotid arteries. The initial examination method is usually duplex sonography. It is a non-invasive tool for determining the degree of carotid stenosis and for determining plaque morphology. Its disadvantage is the subjective evaluation of results [1]. Imaging in B-mode in atherosclerotic carotid bifurcation, the plaque contains calcium and it prevents the passage of an ultrasound wave. Because of this, we can't obtain any information about the vessel below the calcified

\footnotetext{
*Corresponding author: darina.jasikova@tul.cz
} 
segment. This phenomenon is called acoustic shielding. These problems are largely overcome by a combination of B-mode and Doppler ultrasound [2]. In Doppler ultrasonography, the sensor doesn't distinguish blood flow velocity in the middle of the carotid and close to the walls of carotid [3].

Another method is magnetic resonance angiography (MRA). Non-contrast MRA 2D Time of Flight (TOF) is more sensitive to slower flow, and 3D TOF displays a wide range of velocities and has greater accuracy in defining carotid artery morphology. MRA is noninvasive, provides an unlimited number of carotid projections, can evaluate lesions inaccessible to ultrasound, and can detect specific plaque components [2]. The disadvantages of MRA are relatively long data collection, poor resolution and inability to use for routine clinical examination [3].

Computed tomographic angiography is used for its high sensitivity in the evaluation of carotid disease. It provides information about the degree of calcification of the carotid wall and wall plaque. It is wide accessible and non-invasive. We get more information about the composition of the plaque from it. It's accurate in the identification of calcified plaque, but less reliable in describing the morphology of carotid plaque. The carotid lumen is digitally calculated from the entire scanned images of arteries. Because of artefacts from dental amalgam, it cannot be used without a contrast agent to evaluate flow dynamics [2].

The only method that can be used to diagnose dynamic flow is digital subtraction angiography. This is an invasive method, but provides high-quality image that is objective and easy to interpret. It also provides accurate information about the lumen of the carotid artery, but doesn't provide information about plaque composition, vessel walls, or surrounding structures. There is also risk of renal insufficiency and TIA [2].

The trend in investigation of the fluid dynamics is in CFD numerical simulations of the flow in bifurcation using mathematical models [4]. Although many models detailing various anomalies and flow behaviour in bifurcations are created, the experimental verification is still lacking. The design and construction of the experiment is demanding, both in terms of the production of the model, the selection of a suitable methodology for determining the required characteristic physical quantities that can be quantified, as well as adherence to the physiological parameters of the liquid, i.e. working with a phantom resembling real blood. Validation of numerical simulations using the PIV method was dealt with by the authors Sherman in 2014, Liou in 2007, and Hoi in 2006 [5-7]. The main disadvantage of previously made experiments was the preparation of very simplified models. Basically, it was a simplification to the basic geometric shapes, i.e. spheres, cylinders. With the development of 3D scanning techniques and $3 \mathrm{D}$ printing, it is now possible to transform real shapes of human's organs into realistic physiological models. Also, the development of various silicone compositions had seen a significant shift and today we can choose from materials of adequate hardness and surface treatment towards hydrophilic surface characteristics, as is the case with real organs.

\section{Experimental setup and methods}

The aim of the work is to design two anomalies, one with a partially closed stenosis and the other with an aneurysm, and to create their models and silicone castings. The next aim is to build an experimental setup with measuring and synchronizing elements using a pulsatile flow source. Furthermore, based on the measured velocity profiles, calculate the local shear stress and evaluate the results. In their creation, real measurements using Doppler ultrasonography are used as input conditions.

There is a presumption of change in phantom blood flow and the occurrence of vortex structures in the anomalous carotid bifurcation. We assume an accelerated flow compared to the physiological carotid in the aneurysm, and a reduced flow through the affected 
branch is expected at the same time. The change in flow corresponds to a change in pressure in the stenotic branch of the carotid artery. We are dealing with the measurement of dynamic quantities, so the pressure acting on the carotid wall is not derived from the basic relationship for the ratio of static velocity to static pressure. These quantities cannot be measured in vivo on the patient, so a model and laboratory method is used to derivate the pressure near the wall.

\subsection{Carotid artery bifurcation model}

As mentioned in the Introduction, stenosis most often occurs near bends and bifurcations of arteries and is a common cause of stroke. Dynamical forces determined by arterial geometry are keys in the early development of atherosclerotic changes. Novel approach to the study examining carotid flow is by using the PIV method. A solid bifurcation model was created using $3 \mathrm{D}$ printing, from which a silicone rubber model was casted.

The process of model production is more or less standard. After the 3D scan, the data is processed by subsequent modification into the CAD model. CAD model is printed by $3 \mathrm{D}$ printer material, which allows further processing and mainly dissolution in non-aggressive reagent. This form is a negative of the resulting bifurcation. The blood vessel negative is then treated with a coating to prevent the formation of bubbles. This coating also homogenizes and stabilizes the negative surface. Subsequently, the negative is covered with transparent material. After solidification of the material, the negative is removed by dissolution. The resulting model is fitted with connectors and connected to the hydraulic system. Sylgard 184 silicone is still the main material for the production of perfect castings. However, when sclerotic plaques occur, the carotid wall hardens significantly and this modification can be represented with an epoxy resin. Although silicone castings are very popular for ease of manufacture, their surface properties are not physiological because of slightly real organs hydrophilic. For this reason, hydrophilic character is closer to the surface properties of epoxy resin material (Contact Angle (water) 59 ${ }^{\circ}$, Hardness 78 Shore D), although they differ in hardness.

a)

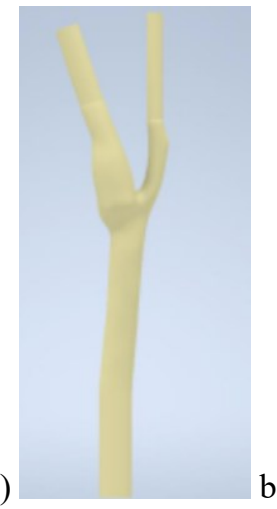

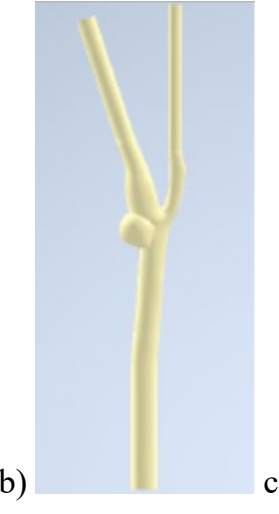

c)

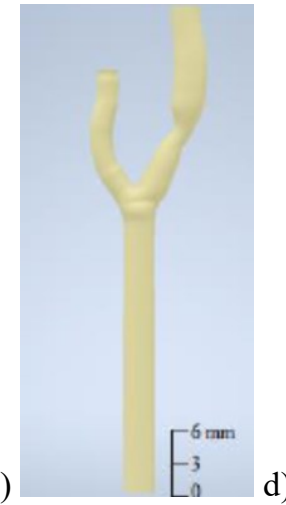

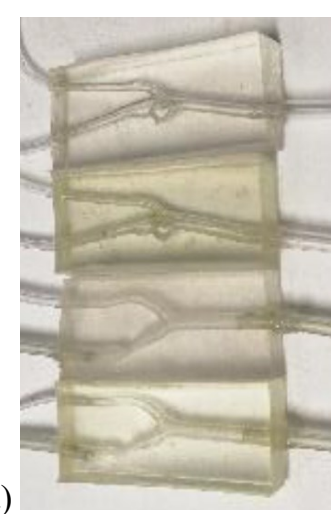

Fig. 1. 3D negative model of: a) physiological carotid artery, b) carotid artery with aneurysm, c) carotid artery with $50 \%$ stenosis of ICA, and d) fitted castings.

\subsection{Experimental PIV Setup and Flow Circuit}

First, an experimental line consisting of a physiological carotid sample, a DMP 331 pressure transducer, a GB P23 PVS micropump, a B.I.O-TECH flow meter and a solenoid valve was assembled (Figure 2). In this line, a pulsation flow with a frequency of $1 \mathrm{~Hz}$ was 
set using a Rigol DG 1022 pulse generator. The excitation pulse lasted on positive level $20 \%$ of cycle duration. The micropump was powered by $13 \mathrm{~V}$, which corresponds to a continuous flow rate of $13 \mathrm{ml} / \mathrm{s}$, and this is the maximum flow through the common carotid. This corresponds to maximal flow rate with opened solenoid valve.

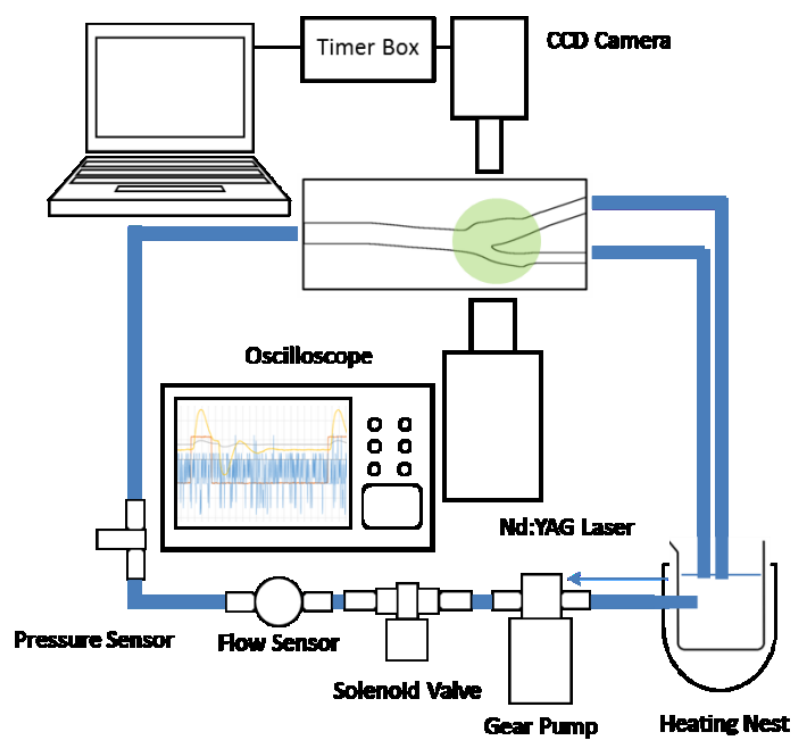

Fig. 2. Schema of experimental setup.

After verifying the functionality of the hydraulic circuit, it was filled with a phantom of blood. The phantom blood was the non-Newtonian liquid solution that contained $900 \mathrm{ml}$ of water, $95 \mathrm{ml}$ of glycerol and $5 \mathrm{~g}$ of dextran [8]. The pressure was adjusted to approximately a physiological value (Figure 3). Change in hydrostatic pressure was adjusted by regulating the level, which was practically done by raising the reservoir to a defined height and reducing the flow through a wider branch of the carotid artery. The pressure was set to $127 / 61 \mathrm{mmHg}, 127 \mathrm{mmHg}$ is the maximum value and $61 \mathrm{mmHg}$ is the minimum value. The mean pressure was $89.7 \mathrm{mmHg}$. The common arterial pressure is approximately $120 \mathrm{mmHg}$ for systole and $70 \mathrm{mmHg}$ for diastole. In arteries with a radius of $0.5 \mathrm{~cm}$, the mean pressure is $90 \mathrm{mmHg}$ [1]. The maximum and minimum values are not ideal, but the mean pressure has been set correctly. At the same setting (liquid inlet parameters), the maximum pressure value for stenosis increased slightly to $128 \mathrm{mmHg}$ and the minimum value decreased to $45 \mathrm{mmHg}$. The mean pressure dropped to $81.3 \mathrm{mmHg}$. For the aneurysm, the maximum value increased to $143 \mathrm{mmHg}$ and the minimum value to $76 \mathrm{mmHg}$. The mean pressure increased to $102.7 \mathrm{mmHg}$. 


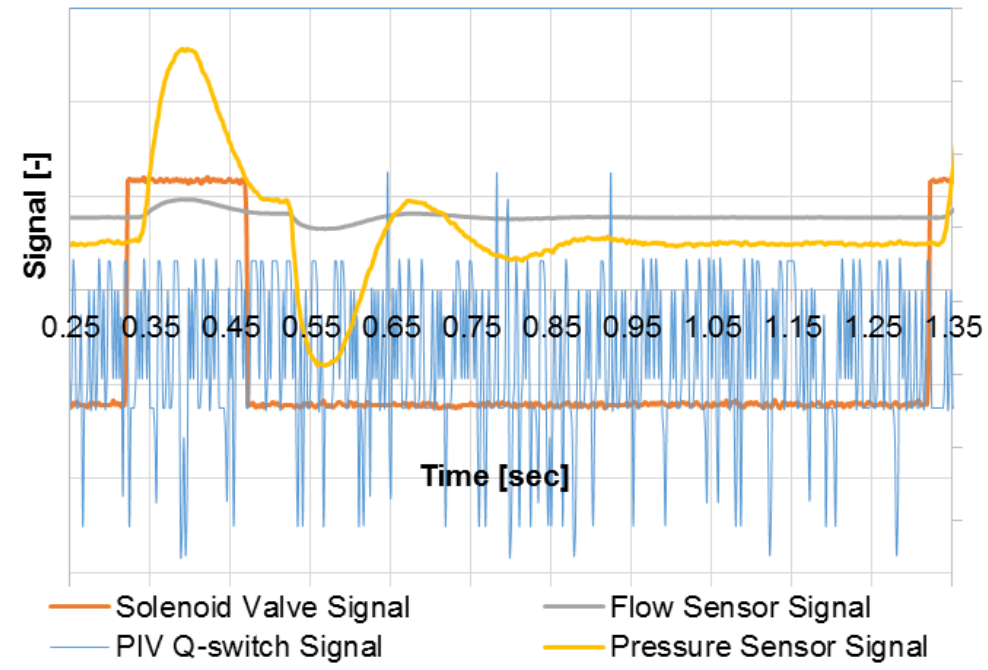

Fig. 3. Recording of the evaluation of individual signals from the oscilloscope.

a)

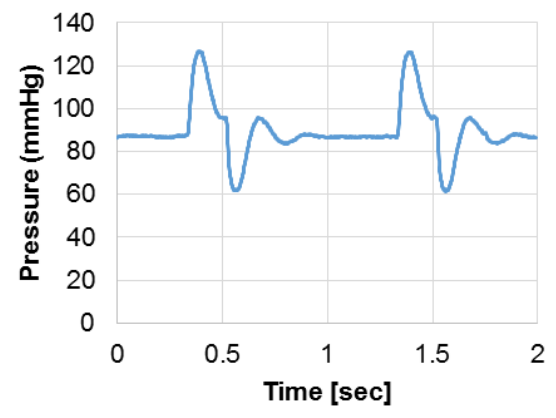

b)

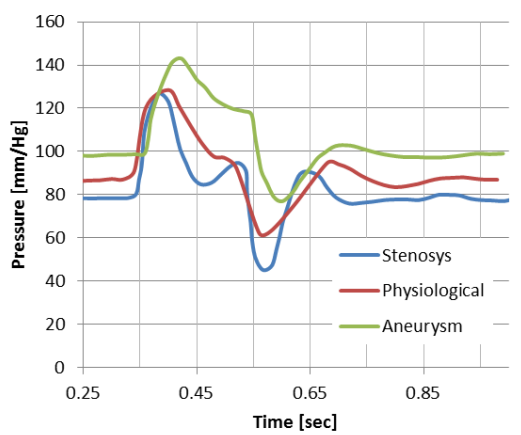

Fig. 4. a) The evaluation of pressure during one excitation pulse after optimal setting of regulators, b) pressure courses for stenosis, physiological, and aneurysm carotid artery.

\subsection{PIV system}

The PIV system consists of a timer box connected to a computer, a NewWave Gemini PIV laser system and a HiSense Neo CCD camera. The sync pulse was outputted from the pulse generator. The pulse lasted $1 \mathrm{~s}$, which corresponds to a resting heart rate. The solenoid valve was opened by switching the $5 \mathrm{~V}$ signal with a leading edge and closed by a falling edge to $0 \mathrm{~V}$. Phantom blood was seeded with polymethylacrylate fluorescent particles coated with Rhodamine-B having a diameter of $10 \mu \mathrm{m}$. As the particles adhered to the surface of the model, Tween surfactant was added to the working fluid in an amount of $6 \mathrm{ml}$ per 11 .

Prior to the measurement, the liquid was heated to $37^{\circ} \mathrm{C}$, which corresponds to the peripheral temperature. The heating of the working fluid took place in a heating nest with precise regulation. Heat losses in the hydraulic circuit were minimal with respect to the velocity of the flowing fluid and the amount in the reservoir. The change in temperature significantly affects the viscosity value, which is a major contributor to the change in the 
nature of the local velocity, and the local change in pressure. Software, Dantec Dynamic Studio, was used to record the data images, in which the images were also subsequently processed. The recording frequency reached $10 \mathrm{~Hz}$ and 250 images of each sample were taken.
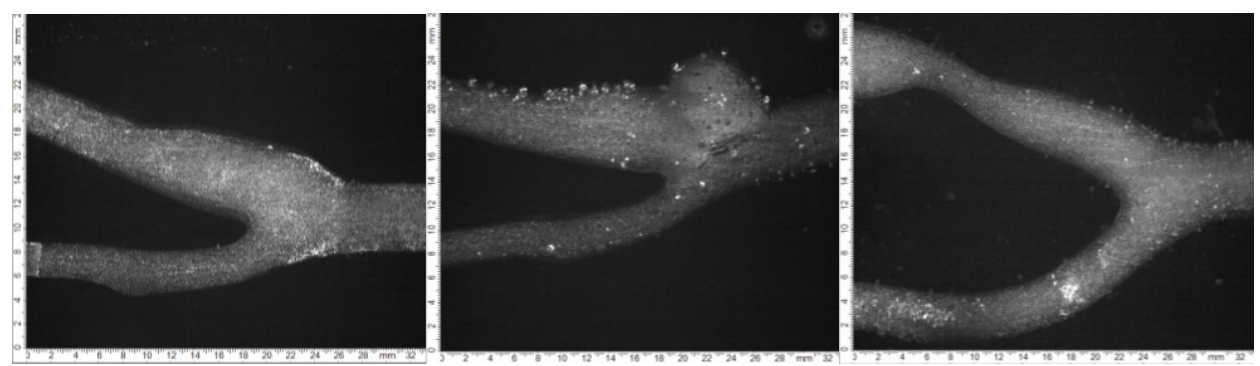

Fig. 5. Camera recording for PIV processing: a) physiological carotid artery, b) carotid artery with aneurysm, c) carotid artery with $50 \%$ stenosis of ICA.

\section{Results and Discussion}

The nature of flow in experimental fluid mechanics is often expressed by ratio numbers. We are dealing with full scale physiological model with real flow rates, so no Re number as levelling crutches is necessary. Here we cite the Reynolds number for the purpose for concept of flow pattern. The hemodynamic forces are determined by vascular geometry. The investigation of model brings quantification and correlation with velocity distribution defined by global geometric parameters: bifurcation angle, planarity, tortuosity and flow character in ICA (internal carotid artery), ECA (external carotid artery), and CCA (common carotid artery). The Reynolds number is in the range between $1425-2137$ according the inner diameter of input part of carotid arteria, i.e. between $6-9 \mathrm{~mm}$.

The significance of the Reynolds number can be challenged from a physical point of view from the essence of the viscosity parameter, which is dependent on temperature and dynamic change. The Reynolds number in the context of pulsatile flow is an irrelevant quantity. Therefore, a dimensionless quantity known as the Womersley number is used for pulsatile flows. Knowledge of the Womersley number is especially important for experimental measurements modelling dynamic processes in body fluid mechanics. It is used to maintain the dynamic similarity in the event of a sudden change in the conditions of the experimental measurement. The Womersley number expresses the relationship between the effects of viscosity and pulsating flow frequency. It is given by the following relation:

$$
\alpha=L \sqrt{\frac{2 \pi f . \rho}{\mu}}
$$

where $\omega$ is angular frequency, $f$ is the ordinary frequency, $L$ is an appropriate length scale (radius of a pipe), $v$ is fluid kinematic viscosity, $\mu$ is fluid dynamic viscosity.

When $(\alpha<1)$, there is developed a typical parabolic velocity profile during each cycle, and there will be given to a good approximation by Poiseuille's law, using the instantaneous pressure gradient. The $(\alpha>10)$ is corresponding with turbulent zone. This determination takes into account time averaging, but if we have a pulsatile flow, the velocity profile is different with respect to course of pressure. This condition is corresponding with turbulent zone that can be expected in a reaction on pressure wave. In our experiment, according to eq. 1 , the radius of CCA was $6 \mathrm{~mm}$, working with dextran solution of density $1.02 \mathrm{~g} / \mathrm{cm}^{3}$ and 
viscosity $4.4 \mathrm{mPa} . \mathrm{s}\left(37^{\circ} \mathrm{C}\right)$, and the angular frequency $2 \pi f$ for $f=1 \mathrm{~Hz}$, the Womersley number is 2.88 . This confirms the dynamic similarity of our experimental model.

The determination takes into account time averaging, but if we have a pulsatile flow, the velocity profile is different with respect to time evaluation of pressure. Here it is important to note that Womersley number expresses a relationship pronouncing two systems of different diameters (radius), fluid properties, and pulsatile flow that could be compared. During the whole experiment, the maximum and minimum values of pressure, synchronization pulse and pulse from the PIV system were monitored on the oscilloscope. Pulsatile flow rate was measured. The continuous flow was generated by a gear pump and the pulsations, as mentioned above, were generated by a pulse generator and a solenoid valve.

a)

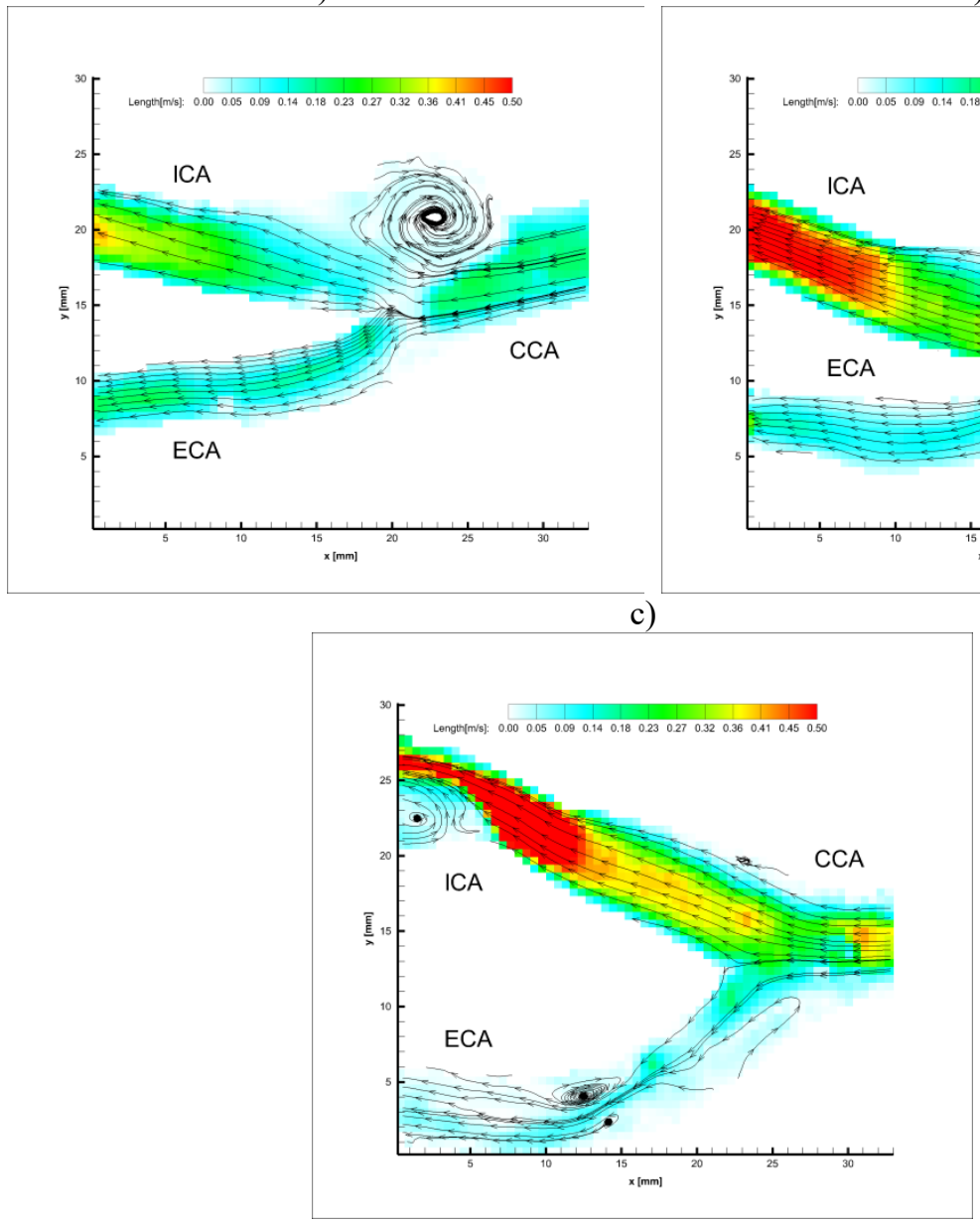

b)

Fig. 6. Camera recording for PIV processing: a) physiological carotid artery, b) carotid artery with aneurysm, c) carotid artery with $50 \%$ stenosis of ICA.

Two second periods with time sampling of $0.002 \mathrm{~s}$ were recorded from the oscilloscope. Due to the large amount of data, they are shown here in graphs, where 1 period is always visible for a specific waveform. The pressure in bar is shown on the minor axis. For the physiological model, the pressure profile, the synchronization pulse and the PIV signal 
were displayed, and for the remaining two, only the pressure profile and the synchronization pulse were displayed.

The processed image data shows the flow in the carotid. Laminar flow is seen in the physiological carotid artery. At the entrance to the model (CCA) it can be seen that in the middle there is a higher flow rate than closer to the walls. This corresponds to the theoretical knowledge that as a result of internal friction, an inhomogeneous vector field of flow velocity is created and creates a so-called velocity parabola. When entering the wider branch of the carotid artery (CCA), the flow slows down slightly due to the carotid bulb and behind it the flow accelerates again. The flow through the narrower branch of the carotid artery (ECA) is significantly slower. The average speed of the physiological model is $0.065 \mathrm{~m} / \mathrm{s}$. The maximum values are around $0.55 \mathrm{~m} / \mathrm{s}$.

In the model with the aneurysm, turbulent flow occurs in the bulge and, as a result, there is an overall reduction in flow rate. The highest flow rate is towards the end of the wider carotid branch. In contrast to the physiological model, the velocity in both branches is similar and there is no noticeable deceleration at the walls. The average flow velocity is $0.035 \mathrm{~m} / \mathrm{s}$. The average speed is much lower than in the physiological model. The maximum speed is around $0.4 \mathrm{~m} / \mathrm{s}$. The shear stress is generally lower than in the physiological model, but it is distributed similarly. It is highest at the top wall of the inner carotid and lowest at the bottom wall. Interestingly, while along the walls of the branches the shear stress has relatively constant dimensions; it varies along the wall of the bulge. This is probably due to the turbulent flow in the bulge. (Figure 6 a)).

In the model with stenosis, a significant increase in the flow rate at the constriction is seen. As the tube expands again, a higher velocity can be seen in the direction in which the liquid flowed from the stenosis, a turbulent flow is created below this point. The current hits the wall above the stenosis at a relatively high speed. As a result, the endothelium is disrupted in the carotid artery and other diseases are possible. In the narrower branch of the carotid artery, the flow is very slow, which is related to the accelerated flow by narrowing in the wider branch. The average speed is $0.061 \mathrm{~m} / \mathrm{s}$. The average velocity is similar to the physiological model, but the figures show that much higher maximum velocities occur in stenosis, around $0.9 \mathrm{~m} / \mathrm{s}$. (Figure $6 \mathrm{~b}$ )).

Authors gratefully thank to the support of The Institute for Nanomaterials, Advanced Technologies, and Innovation, Department of Physical Measurements, and The Faculty of Health Studies.

\section{References}

1. B. S. Certik, S. Machart, M. Novak, Onemocnění karotid a velkých cév aortálního oblouku (Grada, Praha, 2005)

2. A. F. Aburahma, et al., Noninvasive Vascular Diagnosis (4th ed., Cham: Springer International Publishing, 2017)

3. H. B. Kim, et al., Annals of Biomedical Engineering, 32 (2004)

4. C. Chiastra, et.al., BioMed Eng OnLine 15 (2016)

5. C. P. Sherman, et.al., Australas Phys Eng Sci Med 33 (2010)

6. T. M. Liou, Y. Ch. Li, W. Ch. Juan, Journal of Biomechanics 40 (2007)

7. Y. Hoi, et.al., J Biomech Eng. 128 (2006)

8. A. A. Oglat, et. al, The International Conference of Solid State Science and Technology (ICSSST 2017) IOP Conf. Series: Journal of Physics: Conf. Series 1083 (2018) 